\title{
La polykystose rénale dominante de type 1 est clonale et récessive au niveau cellulaire
}

La polykystose rénale dominante est une affection extrêmement fréquente, affectant environ une personne sur mille. Elle se caractérise par le développement de très nombreux kystes rénaux et hépatiques, débutant à différents moments au cours de la vie et de gravité et d'évolution variables. Le plus souvent, la maladie évolue vers une insuffisance rénale chronique, plus ou moins tardive. La forme la plus fréquente, PKD1, représente $85 \%$ des cas et correspond à un locus situé en $16 \mathrm{p} 13.3$ $[1,2]$. Le gène $P K D 1$ code pour une protéine membranaire de 968 acides aminés dénommée polycystine $[2,3]$. De façon singulière, les kystes touchent moins de $1 \%$ des néphrons et se développent de façon focale, à partir d'un segment tubulaire. Par ailleurs, la diversité de l'expression clinique au sein d'une même famille, et même chez des jumeaux monozygotes, est extrême. Pour comprendre le mécanisme de ces particularités, Qian et al. (Baltimore, MD, USA) ont procédé à une analyse génétique de cellules épithéliales décollées par une solution d'EDTA à partir de kystes rénaux indépendants chez 8 femmes atteintes [4]. Ces cellules ont été génétiquement caractérisées pour le locus $P K D 1$ et pour le chromosome $\mathrm{X}$ inactivé. L'analyse du chromosome X inactivé est classiquement utilisé, depuis plusieurs décennies, pour étudier le caractère clonal de tumeurs ou d'affections variées. En effet, chez la femme, un chromosome $\mathrm{X}$ sur deux est normalement inactivé très précocement au cours du développement, de manière parfaitement aléatoire. Ainsi, lorsque, au niveau d'une lésion, toutes les cellules possèdent le même chromosome $\mathrm{X}$ inactivé, cela signifie que cette lésion est de nature importante des auteurs est justement que $82 \%$ des kystes étudiés sont monoclonaux, la maladie elle-même étant cependant polyclonale en ce que le chromosome $\mathrm{X}$ inactivé est souvent différent dans des kystes distincts d'une même malade. Dans les kystes restants, le caractère polyclonal pourrait provenir de la fusion de deux formations kystiques adjacentes. Une perte d'hétérozygotie a été démontrée dans la plupart des kystes étudiés en utilisant le microsatellite KG8, situé dans la région 3' non traduite de l'ARNm PKD1, et le marqueur polymorphe EJ1, localisé dans les exons 45-46, en situation proximale d'environ $1,6 \mathrm{~kb}$ par rapport à KG8. Les allèles perdus correspondent à l'haplotype normal. Ainsi, le développement d'un kyste dépendrait de deux événements, d'une part la mutation germinale, d'autre part la mutation somatique de l'allèle $P K D 1$ initialement normal. C'est ce deuxième événement qui serait l'étape limitante, expliquant la formation focale des kystes. Mais pour rendre compte des milliers de kystes rénaux dans la polykystose et de leur développement parfois très précoce dans la vie, il faut imaginer un taux très élevé de mutations somatiques dans le gène $P K D 1$. Les auteurs font remarquer que ce gène contient une structure polypyrimidique inhabituelle dans l'intron 21 qui pourrait former une triple hélice et constituer une source endogène d'instabilité génétique. Ils suggèrent également que l'inactivation somatique de l'allèle $P K D 1$ normal pourrait être également l'événement critique dans les polykystoses PKD2 car les produits des deux gènes pourraient interagir pour aboutir à la formation des kystes. Un tel schéma d'un développement lésionnel en deux temps dans lequel une perte d'hétérozygotie somatique vient compléter un déficit hétérozygote germinal, est celui bien établi de la tumorigenèse des cancers congénitaux par altération de gènes suppresseurs de tumeurs, par exemple, le rétinoblastome, la polypose colique familiale, la maladie de von Hippel-Lindau et la tumeur de Wilms [5]. D'ailleurs, la nature de la maladie est peut-être réellement tumorale puisqu'il existe plusieurs exemples de souris transgéniques chez lesquelles l'expression anormale d'un oncogène au niveau des tubules rénaux entraîne la formation de kystes [6]. De plus, on sait que le produit anticancéreux Taxol pourraît être efficace en diminuant la formation de ces kystes $\left(\mathrm{m} / \mathrm{s} n^{\circ} 6 / 7\right.$, vol. 10, p. 735). Selon ce schéma, le gène $P K D 1$, pourrait être assimilé à un "anti-oncogène » $(\mathrm{m} / \mathrm{s}$ $n^{\circ} 3$, vol. 13, p. 411). Le déficit en polycystine, ou la synthèse d'une polycystine anormale, perturberait la différenciation et le contrôle de la prolifération des cellules tubulaires à l'origine des formations kystiques.

A.K.
J.P.G.

1. Bachner L. La chasse au gène de la polykystose rénale autosomique dominante. Med Sci 1990; 6: 904-5.

2. Pirson Y, Chauveau D, Watson M, Zeier M, Breuning M. La polykystose autosomique dominante: progrès cliniques et génétiques. $M e d S c i$ $1997 ; 13: 37-44$.

3. Knebelmann B, Grünfeld J. En route vers la compréhension de la kystogenèse dans la polykystose rénale. Med Sci 1995; $11: 1348$.

4. Qian F, Watnick TJ, Onuchic LF, Germino GG. The molecular basis of focal cyst formation in human autosomal dominant polycystic kydney disease type 1. Cell 1996; 87: 979-87.

5. Junien C. Un gène candidat pour la tumeur de Wilms. Med Sci 1990; 6 : 464-9.

6. Trudel M, D'Agati V, Costantini F. c-Myc as an inducer of polycystic kidney disease in transgenic mice. Kidney Int 1996; 39 : 665-71. 